\title{
APRENDIZAJE-SERVICIO Y SU EFECTO SOBRE LAS ACTITUDES HACIA LA INCLUSIÓN EN FUTUROS MAESTROS DE EDUCACIÓN FÍSICA
}

\author{
Jorge Abellán \\ Universidad de Castilla-La Mancha
}

\begin{abstract}
RESUMEN: El aprendizaje-servicio es una metodología que ofrece la posibilidad de aprender mientras se presta un servicio a la comunidad. En el presente trabajo, doce estudiantes debían diseñar y dirigir seis horas de clase relacionadas con los contenidos de la asignatura Juegos, Ocio y Recreación, destinados a prestar un servicio a personas que residían en un centro de atención a la discapacidad intelectual con grandes necesidades de apoyo. Los participantes completaron un cuestionario, antes y después de la experiencia, destinado a la evaluación de sus actitudes hacia la inclusión. Asimismo, realizaron reflexiones individuales después de cada una de las sesiones. Los resultados indican que se observa una tendencia a la mejora en las actitudes hacia la inclusión después del programa, algo que coincide con los resultados del análisis de las reflexiones individuales. Se aprecia un cambio en la percepción de los futuros maestros desde la primera a la última sesión práctica. El primer contacto no fue percibido como una experiencia positiva por los participantes. Los participantes han indicado que la motivación y la adaptación de las tareas han sido sus estrategias inclusivas utilizadas. Se sugiere que las características de los destinatarios del programa han determinado este cambio, ya que los estudiantes se han sentido menos preparados a la hora de atender a la diversidad.
\end{abstract}

PALABRAS CLAVE: aprendizaje-servicio, discapacidad intelectual, formación del profesorado.

\section{SERVICE-LEARNING AND ITS EFFECT ON ATTITUDES TOWARDS INCLUSION IN PRE-SERVICE PHYSICAL EDUCATION TEACHERS}

\footnotetext{
ABSTRACT: Service-learning is a methodology that offers students the possibility to learn while providing a service to the community. In this paper, twelve students had to design and teach six hours of tasks related to the contents of the subject about games, leisure and recreation, for people with intellectual
} 
impairments and high support needs. Participants completed a questionnaire before and after the experience, aimed to evaluate their attitudes toward inclusion. They also made individual reflections after each of the sessions. The results indicate that there is a tendency towards improvement in attitudes toward inclusion after the program, something that corroborates the results of the analysis of individual opinions. The first contact was not perceived as a positive experience by the participants. In addition, participants indicate that the motivation and adaptation of the tasks have been their inclusive strategies used to encourage participation. It is suggested that the characteristics of the people that recieved the program have determined this change, since students have felt less prepared when dealing with diversity.

KEYWORDS: Service-learning, intellectual impairment, teaching training.

Recibido: 02/03/2020

Aceptado: 15/05/2020

Correspondencia: Jorge Abellán, Universidad de Castilla-La Mancha, Facultad de Educación, Campus de Cuenca, Edificio Fray Luis de León, Camino Pozuelo, s/n, 16071 Cuenca. Email: jorge.abellan@uclm.es

\section{INTRODUCCIÓN}

El aprendizaje-servicio (en adelante ApS) es un método pedagógico en el que los estudiantes trabajan los contenidos curriculares a la vez que prestan un servicio a la comunidad (Chiva-Bartoll, Pallarés-Piquer y Gil-Gómez, 2018). Como indica Furco (1996), integrar el ApS en los programas formativos ofrece a los estudiantes la oportunidad de aplicar sus conocimientos en situaciones de práctica real, debido a que se considera una metodología basada en la experiencia (Stanton, 1990). Este método se está utilizando activamente en educación superior, como muestran los numerosos estudios de su aplicación en la educación universitaria publicados recientemente (i.e. Mayor, 2020; Sotelino, Santos y García, 2019; Zayas, Gozálvez y Gracia, 2019).

La utilización del ApS se considera como muy relevante para la formación inicial de los profesionales de la educación (Bates, Drits, Allen y McCandless, 2009), como es el caso de los futuros maestros de Educación Física (en adelante EF) incluidos en este trabajo. De acuerdo con Capella, Gil y Martí (2014), entendemos que la aplicación del ApS en EF supone una práctica novedosa, y debido a las características específicas de la materia, eminentemente práctica, la EF es un marco ideal para la utilización de esta metodología.

Existen ejemplos en los que se combina el ApS y la EF. Chiva-Bartoll et al. (2018) muestran que un programa de ApS ha conseguido mejoras en el concepto de personalidad eficaz de un grupo de futuros docentes de EF. El ApS también se ha utilizado en EF con la intención de disminuir la exclusión social de los receptores del servicio. 
Por ejemplo, Vázquez, Liesa y Lozano (2017) utilizaron un programa de recreos cooperativos realizado por futuros maestros de EF con el objetivo de reducir la exclusión social de los participantes, mientras los futuros maestros aprendían competencias transversales de su grado.

En el ámbito de la EF adaptada también se ha utilizado la metodología del ApS. Por ejemplo, Gil-Gómez, Chiva-Bartoll y Martí-Puig (2015) mostraron que un grupo de futuros maestros mejoraron en su forma de entender la diversidad cultural y especialmente la discapacidad al participar en un programa de ApS en el que diseñaban juegos motores y expresivos para niños con necesidades educativas especiales. En este sentido, Roper y Santiago (2014) mostraron que los estudiantes que realizaron un proyecto de ApS y EF junto a niños con discapacidad mejoraron sus actitudes hacia este colectivo.

Los maestros y futuros maestros de EF han mostrado en muchas ocasiones una actitud positiva hacia la discapacidad y su inclusión en las aulas ordinarias (e.g. Block y Obrusníková, 2007), sin embargo, se han presentado como poco preparados para llevarla a cabo de forma adecuada (Hersman y Hodge, 2010; Vickerman y Coates, 2009). La formación inicial de los docentes se ha considerado como escasa en este campo (Valencia-Péris, Mínguez-Alfaro y Martos-García, 2020), lo que sin duda dificulta la atención a la diversidad por parte de los maestros. En la mayoría de las ocasiones se ha detectado que esta falta de autoeficacia, entendida como el nivel de confianza que muestran los maestros para atender e incluir al alumnado con discapacidad durante sus clases de EF (Bandura, 2001), y unas actitudes negativas hacia la discapacidad o la inclusión, tienen que ver con una falta de contacto y conocimiento sobre las características específicas del colectivo. En esta línea, se ha comprobado que aquellos maestros o futuros maestros con mayor formación en EF adaptada y con mayor contacto con alumnos con discapacidad muestran mayores niveles de autoeficacia (Reina, Hemmelmayr y Sierra-Marroquín, 2016) y actitudes más positivas hacia la inclusión (Ahmmed, Sharma y Deppeler, 2012).

Esta idea ha llevado a los investigadores a diseñar programas para la mejora de las actitudes hacia la inclusión. Por ejemplo, Tindall, Culhane y Foley (2016) evaluaron el efecto de un programa de 10 semanas ( 1 hora a la semana) de práctica de actividad física adaptada, en la que compartían práctica junto a niños y jóvenes con discapacidad, en la autoeficacia percibida por maestros de EF en formación. Los resultados tras la intervención muestran que los participantes mejoraron significativamente su nivel de confianza a la hora de atender e incluir al alumnado con discapacidad.

El objetivo del presente trabajo es evaluar el efecto, en las actitudes hacia la inclusión, de un programa de ApS realizado por un grupo de futuros maestros de EF, prestando un servicio de diseño y puesta en práctica de actividades de EF a un grupo de adultos con discapacidad intelectual (DI) residentes en un centro para la atención a la discapacidad intelectual grave (CADIG). 


\section{Método}

\subsection{Participantes}

La muestra se divide en los prestadores del servicio (futuros maestros de EF) y los receptores del servicio (adultos con DI que vivían en un CADIG). Sus características se detallan a continuación:

Prestadores del servicio: 12 futuros maestros de EF (Medad= 21,83 $\pm 2,16$ años) completaron el proceso completo de ApS, así como los cuestionarios y demás elementos de recogida de datos (se incluyen más detalles en la sección de procedimiento). Todos los participantes eran alumnos del Grado de Maestro de Educación Primaria y estaban cursando la mención de Educación Física. La muestra se dividía en 5 mujeres y 7 hombres. El 58,3\% de la muestra declaró haber tenido contacto previo con personas con discapacidad, mientras que el $41,7 \%$ además había participado junto a ellos en actividades físicas y/o deportivas.

Receptores del servicio: 13 personas con DI (rango= 23-58 años) recibieron el servicio (sesiones de EF). Todos ellos residían en un CADIG durante la realización del ApS. Además, presentaban las siguientes características: dos participantes se desplazaban en silla de ruedas, una participante tenía discapacidad visual y otra tenía discapacidad auditiva. La muestra se dividía en 4 mujeres y 9 hombres.

\subsection{Instrumentos}

\section{Datos cuantitativos}

Se estudiaron las opiniones relativas a la inclusión (ORI) utilizando la adaptación al castellano de la escala Opinions relative to Integration of students with disabilities (Antonak y Larrivee, 1995), realizada por Tárraga, Grau y Peirats (2013). La escala ORI, en su versión en castellano (Tárraga et al., 2013), cuenta con un total de 23 ítems distribuidos en 5 factores: Beneficios de la inclusión; Atención generalista vs. atención especialista; Metodología y manejo de la conducta en el aula; Esfuerzo y dedicación del profesorado hacia los estudiantes con necesidades educativas especiales (NEE); y Formación y competencia del profesorado.

\section{Datos Cualitativos}

El alumnado participante debía realizar un diario de experiencias, en el que recogiese sus impresiones después de cada una de las prácticas (3 en total). Este diario tenía forma de reflexiones formalizadas. Se les pedía que escribiesen en torno a dos ideas fundamentales: i) la diferencia entre lo que habían planificado y lo que realmente habían realizado; y ii) la influencia de la realización de este tipo de experiencias en su formación como futuros maestros de EF. La primera idea se enmarca en la categoría aprendizajes de la práctica docente, mientras que la segunda se incluye 
dentro de la categoría beneficios de la función docente. Ambas categorías se habían utilizado previamente en investigaciones similares (Capella et al., 2014; Palos, 2009). Además, se incluyó una tercera categoría, llamada otros aspectos, en las que se incluían referencias a temas, no propuestos inicialmente, pero interesantes a la hora de analizar el impacto del ApS.

\subsection{Procedimiento}

En el marco de un proyecto de innovación docente, el alumnado de la asignatura Juegos, Ocio y Recreación (obligatoria en la mención de EF), perteneciente al tercer curso del Grado de Maestro en Educación Primaria de una Facultad de Educación española, desarrolló un programa de ApS junto a una asociación de personas con DI $(N=13)$. Todas las personas con DI participantes residían en un CADIG, centro para la atención a las personas con discapacidad intelectual grave impulsado por el gobierno de Castilla-La Mancha.

Los participantes debían completar 6 horas de programa formativo de servicio directo, en el que se les pedía que diseñaran tareas para completar 2 sesiones de EF (de 2 horas cada una) relacionadas con los contenidos de la materia (una sesión destinada al trabajo de los juegos de blanco y diana y otra sesión destinada al trabajo de los juegos cooperativos). Además, en la tercera y última sesión, participaron como entrenadores en un torneo de Boccia inclusivo (2 horas), en el que el grupo de personas con DI formaba equipo con alumnos de un instituto que estaban trabajando la Boccia en sus clases de EF (la experiencia analizada desde el punto de vista del alumnado del instituto se encuentra publicada en Abellán, Sáez-Gallego y Carrión, 2018). La experiencia proporcionó a los destinatarios con DI la oportunidad de realizar actividades de EF, algo que no realizan de manera habitual ya que no disponen de medios personales suficientes (i.e. profesionales de la EF).

La actividad de ApS formaba parte del proceso de evaluación continua dentro de la asignatura de Juegos, Ocio y Recreación. Todas las actividades se llevaron a cabo en un plazo de 3 meses dentro del primer cuatrimestre del curso. La función del profesor fue la de gestionar y coordinar la actividad, ofreciendo a los participantes la oportunidad de revisar sus tareas previamente a las sesiones y ofreciendo consejos sobre cómo interactuar con los participantes. Los estudiantes fueron informados de los destinatarios del servicio, indicándoles que eran personas con DI adultas y con comorbilidades asociadas.

El alumnado debía completar el cuestionario sobre las actitudes hacia la inclusión en dos momentos de medida, antes y después de la realización de la experiencia. Además, los alumnos debían realizar una valoración personal de cada una de las tareas ( 3 en total). Las tomas de datos se llevaron a cabo en lugares tranquilos y con una duración aproximada de 20 minutos. El protocolo utilizado en este estudio está de acuerdo con las exigencias de la Declaración de Helsinki y se encontraba aprobado dentro del protocolo del proyecto de innovación docente. 
Teniendo en cuenta lo expuesto anteriormente, entendemos que la actuación cumple con los requisitos para ser considerado como ApS (de acuerdo con Capella et al., 2014), ya que existe un papel del profesor como guía, mientras que son los alumnos los que planifican y dirigen; además los receptores del servicio son un colectivo en riesgo de exclusión; y por último, se ha trabajado la educación en valores (actitudes hacia la inclusión) y los contenidos específicos de la asignatura (juegos de blanco y diana y juegos cooperativos).

\subsection{Análisis de datos}

Se realizó un enfoque de investigación mixto, en el que los datos cuantitativos obtenidos a partir de cuestionarios fueron completados con datos cualitativos obtenidos a partir de las reflexiones individuales escritas realizadas por los participantes. Dicho enfoque mixto ha sido utilizado recientemente para tratar de explicar con profundidad los fenómenos relacionados con el ApS y la atención a la diversidad (CapellaPeris, Gil-Gómez y Chiva-Bartoll, 2019).

En el análisis cuantitativo, se tomaron los valores medios de los factores como variables dependientes y el efecto del ApS como variable independiente. Debido a la escasez de la muestra, se realizaron pruebas no paramétricas: en concreto para comparar el efecto de la intervención se ha realizado la comparación por pares (pre-test vs. post-test) utilizando la prueba de los rangos con signo de Wilcoxon.

Para el análisis de los datos cualitativos se analizaron las reflexiones formalizadas de cada uno de los participantes al finalizar cada una de las tres sesiones de las que se componía el ApS. Se realizó un análisis deductivo del contenido de cada una de las impresiones en función de tres categorías de análisis: aprendizajes de la práctica docente, beneficios de la función docente y otros aspectos. Dichas categorías se habían utilizado previamente en el trabajo de Capella et al. (2014). Para la exposición de los resultados se ha utilizado una perspectiva narrativa, en la que se han combinado las citas textuales de cada una de las categorías con la narración del autor, profesor de la asignatura en la que se realizaba dicha experiencia, y por lo tanto estuvo presente durante todo el proceso y realización de las actividades. De esta forma se sigue lo indicado por Pérez-Samaniego, Devís-Devís, Smith y Sparkes (2011) en cuanto a la investigación narrativa. Dicha perpectiva está siendo utilizada habitualmente en el campo de la inclusión en EF, como por ejemplo en el reciente trabajo de Martos-García y Monforte (2019).

\section{Resultados}

\subsection{Resultado cuantitativos}

Los resultados obtenidos a partir de la administración de la escala ORI se presentan en la Tabla 1. 
Tabla 1. Diferencias en las actitudes hacia la inclusión

\begin{tabular}{|l|c|c|}
\hline & Pre-test & Post-test \\
\hline Factor 1. Beneficios de la inclusión & $3,63 \pm 0,63$ & $3,45 \pm 0,58$ \\
\hline Factor 2. Atención generalista vs. atención especialista & $2,73 \pm 0,42$ & $3,09 \pm 0,87$ \\
\hline Factor 3. Metodología y manejo de la conducta en el aula & $3,02 \pm 0,88$ & $3,10 \pm 0,74$ \\
\hline $\begin{array}{l}\text { Factor 4. Esfuerzo y dedicación del profesorado hacia los } \\
\text { estudiantes con NEE }\end{array}$ & $2,86 \pm 0,82$ & $2,94 \pm 0,84$ \\
\hline Factor 5. Formación y competencia del profesorado & $2,50 \pm 0,87$ & $2,79 \pm 0,76$ \\
\hline
\end{tabular}

La prueba de Wilcoxon no ha arrojado diferencias significativas para $p<0,05$ en función del momento de medida. Sin embargo, se observa una tendencia a presentar mejores valores después de realizar el ApS, es decir, que las actitudes hacia la inclusión mejoraron en todos los factores (excepto en el factor 1 que empeoró ligeramente). Al realizar un análisis ítem a ítem, la prueba de Wilcoxon reveló diferencias significativas en el ítem 18, que corresponde con el factor beneficios de la inclusión, "es probable que los estudiantes con necesidades educativas especiales creen confusión en un aula generalista" ( $Z=-2,280, p=0,023)$. Después de la intervención, los participantes puntuaron significativamente más bajo en ese ítem, mostrando una actitud más negativa hacia la inclusión.

\subsection{Resultados cualitativos}

Con el objetivo de comprender de forma más profunda las vivencias del alumnado participante, este apartado refleja el resultado del análisis cualitativo de las reflexiones formalizadas de los participantes, que se presentan divididas en las tres categorías de análisis. Al final de cada reflexión se indica, en primer lugar, el número del participante (seguido de P-), y, en segundo lugar, el número de sesión (seguido de $\mathrm{S}$-) en la que realizó dicha reflexión. En las citas literales se han añadido palabras o informaciones extra por parte del investigador, entre corchetes, con el objetivo de ayudar a comprender al lector posibles omisiones de información en la redacción de las reflexiones por parte de los participantes.

\section{Aprendizajes de la práctica docente}

En primer lugar, la mayoría de los participantes destacan la primera impresión cuando comenzó a desarrollarse la primera sesión dentro del ApS:

Antes de la actividad, el profesor nos comentó que íbamos a trabajar con adultos con dificultades. Yo me imaginé en primera instancia una sesión sencilla en la que haríamos de meros guías (...) la idea con la que salí de aquella sesión fue distinta (P5-S1).

El desconocimiento y la falta de experiencia junto a personas con discapacidad se hace patente en los comentarios de los participantes: 
Cuando me informaron que haríamos sesiones con una asociación de personas con discapacidad no tenía ninguna idea de las características de esas personas. (P10-S1). Nunca había trabajado con personas con este tipo de problemas y por eso (...) no podía imaginar que fuera tan difícil. (P4-S1).

Las características de las personas que recibían el servicio (adultos con DI con grandes necesidades de apoyo) hicieron que los estudiantes experimentaran que no habían preparado de manera adecuada sus tareas:

Cuando nos encontramos a las personas a quién iba dirigida la actividad, me di cuenta de que lo que habíamos preparado no podía hacerse en la mayoría de los casos (P2-S1).

El efecto fue un desconcierto y un sentimiento de incomodidad durante la realización de las tareas, que se manifiesta en las reflexiones de los participantes:

Había ciertas situaciones que escapaban a nuestro control o que no sabíamos cómo reaccionar (P3-S1).

Me sentí un poco agobiada ya que no sabía ni donde mirar, ni a quién explicar (P4-S1).

El resultado fue que, al final de la tarea, los participantes no tuvieron una experiencia positiva:

Fue realmente más difícil de lo que me imaginaba (...) me llevé una sorpresa y al menos la primera toma de contacto no me agradó mucho. No disfruté mucho la sesión ya que fue complicada (P5-S1).

Sin embargo, habían surgido en los participantes dos ideas, que se mantendrían durante todo el proceso de ApS: la necesidad de adaptar las tareas a las características específicas de los destinatarios y tratar de motivarlos hacia la práctica:

Nos dimos cuenta de que había que adaptar esos juegos (P9-S1).

No bastaba una explicación sino la repetición (...), la demostración y mucha motivación (P6-S1).

La segunda actividad consiguió que los estudiantes superaran su primera impresión, que no fue muy agradable, ya que conocían a los destinatarios, y sus características, previamente:

Ya tienes más referencias que en la primera sesión (...) ya conoces sus nombres (P1-S2).

En dicha sesión, los futuros maestros se sintieron más preparados y relajados para atender a la diversidad, lo que derivó en un desarrollo más adecuado de la sesión:

No tuvimos que modificar nada de lo que habíamos planteado (P4-S2).

Me he sentido más a gusto con la gente de [nombre de la asociación] (P1-S2).

Fue un gran día ya que personalmente empecé a ver las satisfacciones que se puede llevar un profesor al ver que los alumnos trabajan correctamente (P8-S2). 
Dicha evolución se materializó en la tercera y última sesión, en la que los participantes expresaron sus impresiones sobre su evolución:

He notado un avance grande por nuestra parte a la hora de interactuar con ellos (P1-S3). Mi pensamiento desde la primera toma de contacto hasta la última sesión varió mucho (P5-S3).

Se hace evidente el paso de los seminarios y el resto de las prácticas (...) han propiciado que sea más sencillo afrontar esta etapa (P3-S3).

Además, expresaron que el diseño y la dirección de las tareas había sido realizada acorde a las características de los destinatarios, con la idea inicial de adaptación y motivación a la práctica:

Esta última tarea que hemos preparado ha sido en la que más hemos logrado atraer la atención y motivar al grupo (P2-S3).

Al conocer previamente las características de los participantes estábamos en condiciones de proponer situaciones adecuadas a las necesidades de cada uno (P6-S3).

Por tanto, queda patente que el contacto extendido en el tiempo y la interacción con personas con DI ha hecho modificar su primera impresión negativa hacia una idea de mayor competencia docente.

\section{Beneficios de la función docente}

En este apartado se recogen las impresiones de los participantes cuando reflexionan sobre el impacto del ApS en su formación como futuros maestros de EF. En primer lugar, se exponen las opiniones de los participantes sobre la primera sesión y su impacto sobre su futura profesión docente:

Esta práctica me ha hecho pensar en mi futuro como profesora, ya que una clase de niños puede ser muy diversa (P4-S1).

No solo a mí me ha cambiado (...) mi forma de pensar [tenía experiencia previa en el trabajo junto a personas con discapacidad] (...) si no que [para] todos mis compañeros habrá sido un punto de inflexión (P1-S1).

Además, a partir de la primera sesión, los estudiantes manifiestan un cambio en su forma de explicar las tareas en EF, valorando las capacidades y posibilidades de práctica de todas las personas independientemente de sus limitaciones:

Es muy importante ser claro y conciso, adaptar las reglas cuando sea necesario (P4-S1). Me he dado cuenta con esta práctica que los alumnos con alguna discapacidad pueden realizar las mismas actividades y tareas que el resto del alumnado, aunque en ocasiones han de ser adaptadas a sus características (P9-S1).

Finalmente, esta primera sesión provocó en los participantes una vertiente crítica, especialmente en lo que se refiere a la formación del profesorado para la atención a la diversidad: 
Habría que trabajar más con personas así ya que en cualquier momento nos podemos encontrar con un niño de esas características en una clase, y [para] poder ayudarle de la mejor posible (...) es practicando cuando se aprende. (P10-S1).

Esta crítica al modelo de formación del profesorado también aparece en la tercera sesión:

Bajo mi punto de vista como futuros maestros, si vamos a tener que tratar con alumnado con necesidades educativas especiales, veo una gran deficiencia en ese ámbito educativo que no debería haber (P1-S3).

En la segunda sesión, igual que en la categoría anterior, también se produce un avance positivo en la interpretación del ApS como importante en la formación del profesorado de EF:

Puedo decir que me quedé con un buen sabor de boca ya que había avanzado tanto en mi conocimiento como en mi labor como futuro maestro de EF (P8-S2).

Además, los estudiantes reflexionan sobre el papel del maestro como factor relevante para llevar a cabo una educación inclusiva:

La actitud del profesor es fundamental en estos casos, es decir, una actitud positiva hacia las prácticas [junto a personas con discapacidad] favorece el proceso (P7-S2).

La vertiente crítica despertada en los estudiantes se manifiesta en la reflexión de la última sesión, que muchos participantes utilizan como reflexión final del ApS, describiendo sus aprendizajes como futuros maestros de EF:

Poco a poco hemos ido uniendo las piezas del puzle y creo firmemente en la gran evolución vista desde la primera práctica (...) a la última (...) del desastre al orden, de los nervios a la seguridad. Hemos aprendido a cómo organizar y optimizar nuestras sesiones, a cómo dirigirlas, cómo afrontar los fallos y solucionarlos manteniendo la calma (P3-S3).

He de decir que ha sido una gran experiencia que me ha servido sobre todo para madurar, ya que en cierto modo te abre los ojos (P8-S3).

Hemos trabajado con muchos alumnos con discapacidad a la vez y el que los ejercicios planteados hayan salido bien [en la tercera sesión] me hace pensar que (...) cuando tenga una clase de Primaria sabré actuar [correctamente] si algún alumno tiene discapacidad (P9-S3).

Se puede conseguir que todas las personas las puedas llevar a cabo [las tareas en EF] a partir de una serie de variantes (...), los maestros (...) vamos a tener diferentes alumnos que tengan alguna dificultad en el aprendizaje, y más en $\operatorname{EF}(\ldots)$, al final todos somos iguales y debemos tener las mismas oportunidades para poder llevar a cabo las cosas (P3-S3).

Consecuentemente, ha quedado de manifiesto que el ApS ha conseguido que los estudiantes se planteen su futuro, la necesidad de adaptar su docencia a las características de los alumnos y la necesidad de que todos sus alumnos tengan igualdad de oportunidades. 


\section{Otros aspectos}

En este apartado se incluyen aquellos aspectos relevantes que no han sido incluidos en ninguno de los dos apartados anteriores. En primer lugar, muchos alumnos refieren que la experiencia es un claro ejemplo de educación en valores y de superación de dificultades.

Me han hecho ver que por muchas discapacidades que tengamos si queremos podemos (P7-S1).

Me han demostrado que pese a todos los inconvenientes que te puede presentar la vida, siempre hay que seguir adelante y no cerrarse puertas nunca (P8-S1).

Una experiencia dotada de valores, de integración y de otro punto de vista enfocado a distintos usos aplicables de nuestros conocimientos adquiridos en el grado (P3-S1).

Esta idea de utilizar los conocimientos adquiridos en su formación en otros ámbitos ha hecho que algunos de los participantes se planteen incluso la posibilidad de trabajar en el ámbito de las asociaciones para personas con DI.

Hemos conocido otro ámbito laboral donde trabajar (P3-S3).

Finalmente, los participantes indican que volverían a realizar este tipo de programa, por ejemplo:

Es una práctica que repetiría, ya que los resultados fueron muy gratificantes y ver como todo el mundo se involucraba en su tarea fue lo que más me llamo la atención (P9-S2).

\section{Discusión}

El objetivo de la presente investigación era comprobar el efecto de un programa de ApS en EF sobre las actitudes hacia la inclusión de futuros maestros de EF. Con tal fin se analizaron las actitudes ante la inclusión en EF de los participantes en un programa de ApS consistente en la elaboración y realización de sesiones de EF dirigidas a personas con $\mathrm{DI}$, con gran necesidad de apoyo y comorbilidades asociadas, en las que se trabajaban los contenidos de la asignatura Juegos, Ocio y Recreación, encuadrada dentro de la mención de EF en el Grado de Maestro en Educación Primaria.

En primer lugar, los resultados indican que la experiencia mejoró las actitudes hacia la inclusión en todas las variables analizadas (excepto en el factor beneficios de la inclusión), sin embargo, la ausencia de diferencias significativas entre los momentos de medida (pre-test y post-test) indica que los resultados no son concluyentes. Se observa por tanto una tendencia a la mejora de las actitudes hacia la inclusión tras el ApS, en parte de acuerdo con la teoría del contacto directo (Allport, 1954). Parece que el contacto con personas pertenecientes a un colectivo en riesgo de exclusión social mejora sus actitudes hacia dicho colectivo como ya se había demostrado en investigaciones previas en EF (Molina y Valenciano, 2010; Santana y Garoz, 2013). Esta mejora se hace patente en el análisis cualitativo realizado, ya que los participantes se han sentido cada vez más cómodos a la hora de tratar a las personas con DI, receptoras del servicio, conforme avanzan sus interacciones con ellos. Especialmente 
relevante es el cambio de actitudes manifestado a partir de la primera sesión del ApS que correspondía con el primer contacto con personas con discapacidad de un buen número de los participantes (el 41,7\%).

La impresión después de la primera práctica fue negativa, los estudiantes no esperaban tener que tratar con personas con DI con un alto grado de necesidad de apoyo, por lo que se vieron desbordados por la situación, como se ha indicado en el análisis cualitativo, especialmente al referirse los estudiantes a las diferencias entre lo que habían planificado y lo que realmente habían podido realizar. Parece que este primer contacto no cumplió con lo indicado por Sherrill (1993), que propone que para modificar las actitudes se deben establecer situaciones placenteras, y no sensaciones de agobio, como las manifestadas por los estudiantes. En futuras investigaciones se sugiere que los futuros maestros se enfrenten, al menos en un primer momento, con personas con discapacidad con menos necesidad de apoyo, con las que puedan sentirse algo más competentes y preparados a la hora de atender la diversidad, para con el paso de las sesiones, poder enfrentarse a retos más complejos. Además, los participantes disponían de una formación escasa sobre cómo dirigir sesiones de EF (cursaban su primera asignatura de la mención de EF y la tercera dentro del área de conocimiento) y sobre cómo atender a la diversidad (se encontraban en un momento inicial de su formación -primer cuatrimestre del tercer curso-) y sin formación específica sobre EF adaptada. Por ello, en futuras investigaciones se debería, por un lado, ofrecer más información a los participantes sobre las características de los destinatarios del servicio y, por otro lado, realizar las experiencias más complejas con alumnado de cursos superiores y por tanto más formados. Por otro lado, se asume un posible error en la planificación del primer contacto, que puede ser subsanado en futuras investigaciones estableciendo contactos previos a los prestadores del servicio en los que puedan conocer a los receptores del servicio sin tener que comenzar directamente su intervención en ese momento. Sugerimos la utilización de jornadas de presentación para favorecer el desarrollo de los programas de ApS.

Por otro lado, de acuerdo con lo que indica Deeley (2016), las experiencias que producen en los participantes incomodidad, como es el caso de la primera sesión del programa ApS que nos ocupa, pueden ser fuente de aprendizaje, y sin duda, como indica González-Geraldo (2019), el ApS puede generar esa incomodidad, entendida, al menos en lo que respecta a esta investigación, como ese momento en el que nos damos cuenta que no estabamos totalmente preparados para enfrentarnos a una determinada situación profesional, lo que pone en duda nuestra percepción de competencia.

Este primer contacto negativo también explica los resultados tanto en la pregunta que obtiene un descenso significativo después del post-test ("es probable que los estudiantes con necesidades educativas especiales creen confusión en un aula generalista") en el cuestionario sobre actitudes hacia la inclusión que emana del análisis cualitativo. Investigaciones previas habían demostrado que los futuros maestros suelen presentar actitudes positivas hacia la inclusión (e.g. Abellán y Sáez-Gallego, 2020) pero una baja preparación para atender a la diversidad (Hersman y Hodge, 
2010; Vickerman y Coates, 2009).

Los estudiantes suelen percibir que no han sido suficientemente formados para incluir alumnos con discapacidad en sus clases de EF (Block, Taliaferro, Harris y Krause, 2010). Los resultados del análisis cualitativo están de acuerdo con esta afirmación, ya que los estudiantes reclaman más contacto con personas con discapacidad y en general una mayor formación en EF adaptada. Los participantes han descubierto dos de las mejores estrategias a la hora de fomentar la inclusión en EF: por un lado, la adaptación de las tareas y por otro, el aumento de la motivación. Los participantes también han logrado superar uno de los principales problemas a la hora de enfrentarse al reto de la inclusión, la ausencia de estrategias inclusivas, de acuerdo con Vlachou, Didaskalou y Voudouri (2009). La adaptación de tareas ya había sido referida como una de las estrategias más adecuadas para conseguir la inclusión en EF (Ríos, 2009).

Por último, la realización del ApS ha despertado la perspectiva crítica de los participantes, que se han cuestionado su propia formación y la organización de los programas formativos, con una ausencia notable de formación específica de inclusión, lo que ha demostrado ser determinante para mostrar una preparación adecuada en atención a la discapacidad en EF. Como indica Ríos (2009), esta vertiente crítica es una de las características de un maestro comprometido con la inclusión. Además, otras experiencias de ApS en futuros maestros de EF habían logrado despertar esa vertiente crítica del alumnado participante (Sanz y Ramo, 2015). Por ejemplo, Chambers y Lavery (2012) mostraron que el pensamiento crítico era uno de los aspectos que emergía a partir de las reflexiones y experiencias de futuros maestros que habían participado en experiencias de ApS.

\section{Conclusiones}

Teniendo en cuenta la naturaleza exploratoria del trabajo, lo reducido de la muestra y el objetivo propuesto, se puede concluir que en esta población de estudio, el ApS junto a personas con DI ha supuesto un reto, en parte no conseguido, debido a la ausencia de experiencias previas y de formación en este campo. Sin embargo, los resultados en el análisis de sus actitudes hacia la inclusión y especialmente el análisis de sus reflexiones, hacen patente que se ha producido un aprendizaje sobre la forma de atender a la diversidad en EF, emergiendo la necesidad de motivar hacia la práctica y fundamentalmente la necesidad de adaptar las tareas a las necesidades específicas del alumnado como herramientas que fomentan la inclusión en EF. Adicionalmente, se ha despertado una vertiente crítica en el alumnado, que reclama más experiencias similares y una mayor formación específica en EF adaptada de cara a su futura práctica docente. Los hallazgos de este trabajo, tanto positivos como negativos, deben ser tenidos en cuenta a la hora de diseñar nuevos programas de ApS con poblaciones similares. Es especialmente necesario explorar el efecto del grado de necesidad de apoyo de los grupos de personas con discapacidad receptores de los servicios, y si esa variable puede ser relevante en el efecto de los programas destinados a preparar a los futuros maestros para atender a la diversidad en sus clases. 


\section{REFERENCIAS BIBLIOGRÁFICAS}

Abellán, J. y Sáez-Gallego, N. M. (2020). Opiniones relativas a la inclusión de los alumnos con necesidades educativas especiales mostradas por futuros maestros de infantil y primaria. Revista complutense de educación, 31(2), 219-229. https:// doi.org/10.5209/rced.62090

Abellán, J., Sáez-Gallego, N. M. y Carrión, S. (2018). La boccia como deporte adaptado y sensibilizador en Educación Física en Educación Secundaria. SPORT TK-Revista Euroamericana de Ciencias del Deporte, 7(2), 109-114. https://doi. org/10.6018/sportk.343011

Ahmmed, M., Sharma, U. y Deppeler, J. (2012). Variables affecting teachers' attitudes towards inclusive education in Bangladesh. Journal of Research in Special Educational Needs, 12(3), 132-140. https://doi.org/10.1111/j.1471-3802.2011.01226.x

Allport, G. (1954). The nature of prejudice. New York: Doubleday Books.

Antonak, R. F. y Larrivee, B. (1995). Psychometric analysis and revision of the opinions relative to mainstreaming scale. Exceptional Children, 62, 139-149. https:// doi.org/10.1177/001440299506200204

Bandura, A. (2001). Social cognitive theory: An agentic perspective. Annual Review of Psychology, 52, 1-26. https://doi.org/10.1146/annurev.psych.52.1.1

Bates, A. K., Drits, D., Allen, C. y McCandless, P. (2009). Service learning as an instructional strategy for the preparation of teachers. The Journal of Effective Teaching, 9, 5-23.

Block, M., Taliaferro, A., Harris, N. y Krause, J. (2010). Using self-efficacy theory to facilitate inclusion in general physical education. Journal of Physical Education, Recreation and Dance, 81(3), 43-46. https://doi.org/10.1080/07303084.2010.10 598448

Block, M. E. y Obrusníková, I. (2007). Inclusion in physical education: A review of the literature from 1995-2005. Adapted Physical Activity Quarterly, 24(2), 103-124. https://doi.org/10.1123/apaq.24.2.103

Capella, C., Gil, J. y Martí, M. (2014). La metodología del aprendizaje-servicio en la educación física. Apunts. Educación Física y Deportes, 116(2), 33-43. https://doi. org/10.5672/apunts.2014-0983.es.(2014/2).116.03

Capella-Peris, C., Gil-Gómez, J. y Chiva-Bartoll, O. (2019). Innovative Analysis of ServiceLearning Effects in PhysicalEducation: A Mixed-Methods Approach. Journal of Teaching in Physical Education, 39(1), 102-110. https://doi.org/10.1123/jtpe.2019-0030

Chambers, D. J. y Lavery, S. (2012). Service-learning: A valuable component of preservice teacher education. Australian Journal of Teacher Education, 37(4), 128137. https://doi.org/10.14221/ajte.2012v37n4.2

Chiva-Bartoll, O., Pallarés-Piquer, M. y Gil-Gómez, J. (2018). Aprendizaje-servicio y mejora de la Personalidad Eficaz en futuros docentes de Educación Física. 
Revista Complutense de Educación, 29(1), 181-197. https://doi.org/10.5209/ RCED.52164

Deeley, S. J. (2016). El Aprendizaje-Servicio en educación superior. Teoría, práctica y perspectiva crítica. Madrid: Narcea.

Furco, A. (1996). Service-learning: A balanced approach to experiential education. En B. Taylor (Ed.) Expanding Boundaries: Serving and Learning. Washington: Corporation for National Service, pp. 2-6.

Gil-Gómez, J., Chiva-Bartoll, O. y Martí-Puig, M. (2015). The impact of service learning on the training of pre-service teachers: Analysis from a physical education subject. European Physical Education Review, 21(4), 467-484. https://doi. org/10.1177/1356336X15582358

González-Geraldo, J. L. (2019). El aprendizaje-servicio en educación superior. teoría, práctica y perspectiva crítica. Teoría De La Educación. Revista Interuniversitaria, 31(1), 190-192.

Hersman, B. L. y Hodge, S. R. (2010). High school physical educators' beliefs about teaching differently abled students in an urban public-school district. Education and Urban Society, 42(6), 730-757. https://doi.org/10.1177/0013124510371038

Martos-García, D. y Monforte, J. (2019). 'Haz lo que puedas'. Un estudio de caso sobre diversidad funcional y Educación Física. Ágora para la Educación Física y el Deporte, 21, 52-73. https://doi.org/10.24197/aefd.0.2019.52-73

Mayor, D. (2020). Aprendizaje-Servicio como estrategia metodológica para impulsar procesos de educación expandida. Multidisciplinary Journal of Educational Research, 10(1), 47-74. http://dx.doi.org/10.17583/remie.2020.4562

Molina, J. P. y Valenciano, J. (2010). Creencias y actitudes hacia un profesor de educación física en silla de ruedas: un estudio de caso. Revista de Psicología del Deporte, 19(1), 137-149.

Palos, J. (2009). ¿Por qué hacer actividades de aprendizaje servicio? En J. M. Puig, (Coord.), Aprendizaje servicio (APS): educación y compromiso cívico (pp. 151161). Barcelona: Graó.

Pérez-Samaniego, V., Devís-Devís, J., Smith, B. M. y Sparkes, A. C. (2011). La investigación narrativa en la educación física y el deporte: qué es y para qué sirve. Movimento, 7(1), 11-38. https://doi.org/10.22456/1982-8918.17752

Reina. R., Hemmelmayr, I. y Sierra-Marroquín, B. (2016). Autoeficacia de profesores de educación física para la inclusión de alumnos con discapacidad y su relación con la formación y el contacto previo. Psychology, Society, \& Education, 8(2), 93103. https://doi.org/10.25115/psye.v8i2.455

Ríos, M. (2009). La inclusión en el área de educación física en España. Análisis de las barreras para la participación y aprendizaje. Ágora Educación Física y Deporte, 9, 83-114. 
Roper, E. A. y Santiago, J. A. (2014). Influence of service-learning on kinesiology students' attitudes toward P-12 students with disabilities. Adapted Physical Activity Quarterly, 31, 162-180. https://doi.org/10.1123/apaq.2013-0086

Santana, P. y Garoz, I. (2013). Actitudes hacia la discapacidad e intervención docente desde el deporte adaptado. Revista Internacional de Medicina y Ciencias de la Actividad Física y el Deporte, 49(13), 1-17.

Sanz, I. y Ramo, R. M. (2015). Aproximación a los impactos y beneficios del aprendizaje servicio en la Universidad de Zaragoza. RIDAS: Revista Iberoamericana de Aprendizaje y Servicio, 1, 9-27. https://doi.org/10.1344/RIDAS2015.1.2

Sherrill, C. (1993). Adapted physical activity, recreation and sport: cross disciplinary and lifespan. Dubuke: Mcgraw-Hill.

Sotelino, A., Santos, M. A. y García, J. (2019). El aprendizaje-servicio como vía para el desarrollo de competencias interculturales en la Universidad. Educatio Siglo XXI, 37, 73-90. https://doi.org/10.6018/educatio.363391

Stanton, T. (1990). Service learning: Groping toward a definition. En J. C. Kendall et al. (Coords.), Combining Service and Learning: A Resource Book for Community and Public Service (Vol. 1) (pp. 65-67). Raleigh, NC: National Society.

Tárraga, R., Grau, C. y Peirats, J. (2013). Actitudes de los estudiantes del Grado De Magisterio y del Máster de Educación Especial hacia la inclusión educativa. Revista Electrónica Interuniversitaria de Formación del Profesorado, 16(1), 55-72. https://doi.org/10.6018/reifop.16.1.179441

Tindall, D., Culhane, M. y Foley, J. (2016). Pre-service teachers' self-efficacy towards children with disabilities: An Irish perspective. European Journal of Adapted Physical Activity, 9(1), 27-39. https://doi.org/10.5507/euj.2016.003

Valencia-Peris, A., Mínguez-Alfaro, P. y Martos-García, D. (2020). La formación inicial del profesorado de Educación Física: una mirada desde la atención a la diversidad. Retos, 37, 597-604.

Vázquez, S., Liesa, M. y Lozano, A. (2017). Recreos cooperativos e inclusivos a través de la metodología de Aprendizaje-Servicio. Revista Electrónica Interuniversitaria de Formación del Profesorado, 20(1), 173-185. https://doi.org/10.6018/reifop/20.1.213181

Vickerman, P. y Coates, J. K. (2009). Trainee and recently qualified physical education teachers' perspectives on including children with special education needs. Physical Education and Sport Pedagogy, 14(2), 137-153. https://doi. org/10.1080/17408980802400502

Vlachou, A., Didaskalou, E. y Voudouri, E. (2009). Adaptaciones en la enseñanza de los maestros de Educación General: repercusiones de las respuestas de inclusión. Revista de Educación, 349, 179-202.

Zayas, B., Gozálvez, V. y Gracia, J. (2019). La Dimensión Ética y Ciudadana del Aprendizaje Servicio: Una apuesta por su institucionalización en la Educación Superior. Revista Complutense de Educación, 30, 1-15. https://doi.org/10.5209/RCED.55443 